\title{
Top Most Surface Studies by Total Reflection Positron Diffraction*
}

\author{
A. Kawasuso, T. Ishimoto, Y. Fukaya, and K. Hayashi \\ Japan Atomic Energy Research Institute, Advanced Science Research Center, \\ Watanuki, 1233, Takasaki, Gunma, 370-1292, Japan \\ A. Ichimiya ${ }^{\dagger}$ \\ Japan Atomic Energy Research Institute, Advanced Science Research Center, \\ Watanuki, 1233, Takasaki, Gunma, 370-1292, Japan and \\ Department of Quantum Engineering, Nagoya University, Furocho, Chikusa-ku, Nagoya, 464-8603 Japan
}

(Received 3 October 2003; Accepted 8 November 2003; Published 27 December 2003)

\begin{abstract}
In this article, we detail the development and application of reflection high-energy positron diffraction (RHEPD). A fine positron beam with a coherence length of approximately $170 \AA$ was obtained by the electrostatic lens system. The beam quality is good enough to observe the RHEPD patterns from a clean $\mathrm{Si}(111) 7 \times 7$ and $\mathrm{SiC}(0001)$ surfaces. The rocking curve associated with the $\operatorname{Si}(111) 7 \times 7$ surface revealed the fact that the vertical position of the adatom layer relative to the first layer is sufficiently relaxed towards the vacuum region than that expected from the electron diffraction experiments. After the high temperature hydrogen etching, the SiC surface became atomically smooth. It was found that $\mathrm{Si}$ face is somewhat unstable to the oxygen adsorption as compared to $\mathrm{C}$ face. The rocking curves were reproduced assuming the attachment of oxygen atoms at $T_{1}$ site with a bond length of $1.88 \AA$. Heating the $\mathrm{SiC}$ surface in the ultra-high vacuum after the sacrificial oxidation and hydrofluoric etching, an atomically flat carbon rich surface appeared. [DOI: 10.1380/ejssnt.2003.152]
\end{abstract}

Keywords: Reflection High-Energy Positron Diffraction; RHEPD; Total Reflection; Rocking Curve; $\operatorname{Si}(111) 7 \times 7 ; \operatorname{SiC}(0001)$; Hydrogen Etching

\section{INTRODUCTION}

Taking advantage that positrons are totally reflected at crystal surfaces, reflection high-energy positron diffraction (RHEPD) is a potential tool to study the surface atomic structures [1]. Positron penetration depth is limited to a few $\AA$ in the total reflection mode and hence it is possible to extract information only from the first surface layer with a minimum influence of the interior. Total reflection never occurs in the case of electron diffraction. Although X-rays are also totally reflected at surfaces, the penetration depth is typically $100 \AA$ that is much greater than that of positron. Using the positron total reflection, several surface related phenomena, such as phase transition, soft lattice vibration and surface melting, can be argued more precisely than using the electron and X-ray diffraction methods. In this article, we demonstrate the development of RHEPD and the first observation of the RHEPD pattern from a clean $\mathrm{Si}(111) 7 \times 7$ surface. We also show the application of RHEPD to $\mathrm{SiC}(0001)$ surfaces.

\section{INSTRUMENTATION}

A $20 \mathrm{keV}$ well-collimated positron beam was generated using the electrostatic lenses as shown in Fig. $1[2,3]$. A positron source with an activity of $217 \mathrm{MBq}$ and an active diameter of $5 \mathrm{~mm}$ was installed in the positron gun part. The source was electrically floated at $20 \mathrm{kV}$. The fast positrons were moderated using a well-annealed tungsten single crystal with a thickness of $500 \mathrm{~nm}$. The

*This paper was presented at The 4th International Symposium on Atomic Level Characterizations for New Materials and Devices (ALC '03), Kauai, Hawaii, USA, 5-10 October, 2003.

†Corresponding author: ichimiya@nuqe.nagoya-u.ac.jp

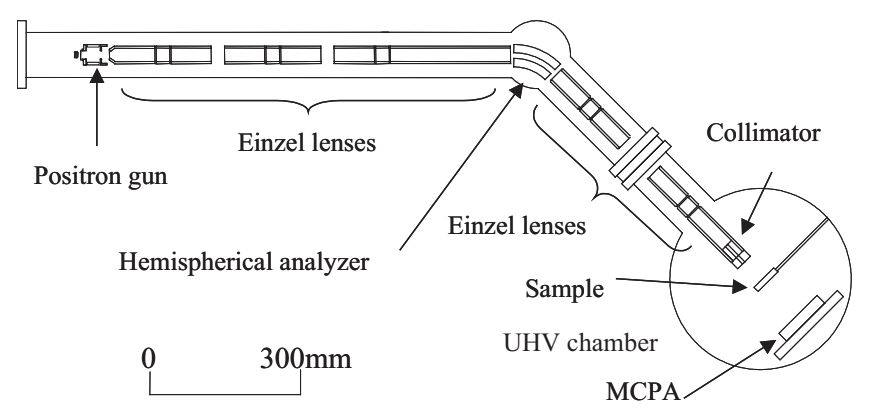

FIG. 1: Schematic overview of the positron beam apparatus.

slow positrons were extracted from the moderator using a grid electrode with a relative bias potential of $-0.4 \mathrm{kV}$. Then, the positron beam is focused by the Wehnelt, Soa and anode electrodes. The bias potential of the anode electrode was set to be $19.2 \mathrm{kV}$ and those for the former two electrodes were adjusted between $19.6 \mathrm{kV}$ and 19.2 $\mathrm{kV}$. The above positron gun was realized by the Brandeis research group [4]. Three unipotential lenses were installed after the anode exit. To prevent the direct fly of non-thermal positrons, a hemispherical type electrostatic analyzer with a bent angle of $45^{\circ}$ was fabricated. Then, the positron beam was further transported using two unipotential lenses. Finally, the beam was deflected downward by $2.8^{\circ}$ and collimated to $1 \mathrm{~mm}$ diameter using a long pinhole slit (100 $\mathrm{mm}$ length). Although the beam flux was rather weak $\left(2 \times 10^{3} \mathrm{e}^{+} / \mathrm{s}\right)$, the energy dispersion and the angular spread were reduced to less than $0.1 \%$ and $0.1^{\circ}$, respectively. The beam characteristics are listed in Table I. So far, the goodness of a beam is expressed in terms of the brightness per volt. Referring to the expression of Brandes [5], the present brightness per volt is estimated to be approximately $\sim 10^{6} \mathrm{e}^{+} / \mathrm{s} / \mathrm{cm}^{2} / \mathrm{rad}^{2} / \mathrm{V}$, which is more or less comparable to the case employing the brightness enhancement technique. Thus, we may not 
TABLE I: Characteristics of the present positron beam.

\begin{tabular}{ccccc}
\hline \hline Source & Energy & $\Delta E$ & $\Delta \theta$ & Flux \\
\hline${ }^{22} \mathrm{Na} / 270 \mathrm{MBq}$ & $20 \mathrm{keV}$ & $20 \mathrm{eV}$ & $0.1^{\circ}$ & $2 \times 10^{3} \mathrm{e}^{+} / \mathrm{s}$ \\
\hline \hline
\end{tabular}

set up the re-moderation stage as long as the restriction from the Liouville' $s$ theorem is reduced by accelerating positrons.

Instead of the brightness per volt, we have to import a concept of coherence length, which is used as the goodness of a beam in diffraction experiments, as follows [6]. Any beams have energy spreads $(\Delta E)$. Therefore, the incident beam should be described as a wave packet and not as a plane wave. This means that the beam has a finite wavelength. If the size of the surface unit cell is larger than the wavelength, the surface super structure is hardly observed as fractional order spots. The probability amplitude of the plane wave is uniform everywhere and thus there are no limitations in the observable unit cell size. The coherence length in the surface parallel direction is given by $l_{x}=2 \pi / \Delta k=24.5 E^{1 / 2} / \Delta E$, where $\Delta k$ is the spread of the wave number. Due to a similar reason, the angular divergence of the beam $(\Delta \theta)$ regulates the coherence length to the surface vertical direction: $l_{y}=12.3 / \Delta \theta / E^{1 / 2}$. A typical example to test the coherence length of the beam is the $\operatorname{Si}(111) 7 \times 7$ surface which has seven times greater lattice constant $(=3.84 \AA$ $\times 7 \sim 30 \AA)$ as compared to the bulk truncated surface. Thus, to observe the Si- $7 \times 7$ surface the energy spread should be reduced to less than $\sim 100 \mathrm{eV}$ when $E=20 \mathrm{keV}$.

A $20 \mathrm{keV}$ positron beam was reflected at the surface at a glancing angle of $0.5-4.2^{\circ}$. Reflected positrons were observed using the microchannel plate assembly with a phosphor plane (Hamamatsu F2226-24P). Phosphor plane images were digitally accumulated until adequate brightness was achieved. Auger electron spectroscopy measurements were also carried out to check the surface contamination level and to obtain the compositional information.

\section{EXPERIMENTS, RESULTS AND DISCUSSION}

\section{A. $\quad \operatorname{Si}(111) 7 \times 7$}

Nowadays, the structure of $\operatorname{Si}(111) 7 \times 7$ surface is almost fully understood. Takayanagi et al. proposed socalled dimmer-adatom-stacking fault (DAS) model based on the Patterson map of the transmission electron diffraction and scanning tunneling microscopy observation $[7,8]$. Interlayer distances of the DAS structure have been determined using low energy electron diffraction (LEED) [9], reflection high-energy electron diffraction (RHEED) $[10,11]$ and X-ray diffraction (XRD) $[12]$. A critical discrepancy on the adatom vertical position is known to appear between electron diffraction and XRD. That is, the adatom vertical position from the first layer determined using XRD is $0.2 \AA$ greater than that determined using electron diffraction. To determine the adatom vertical position, we examined the $\operatorname{Si}(111) 7 \times 7$ surface using

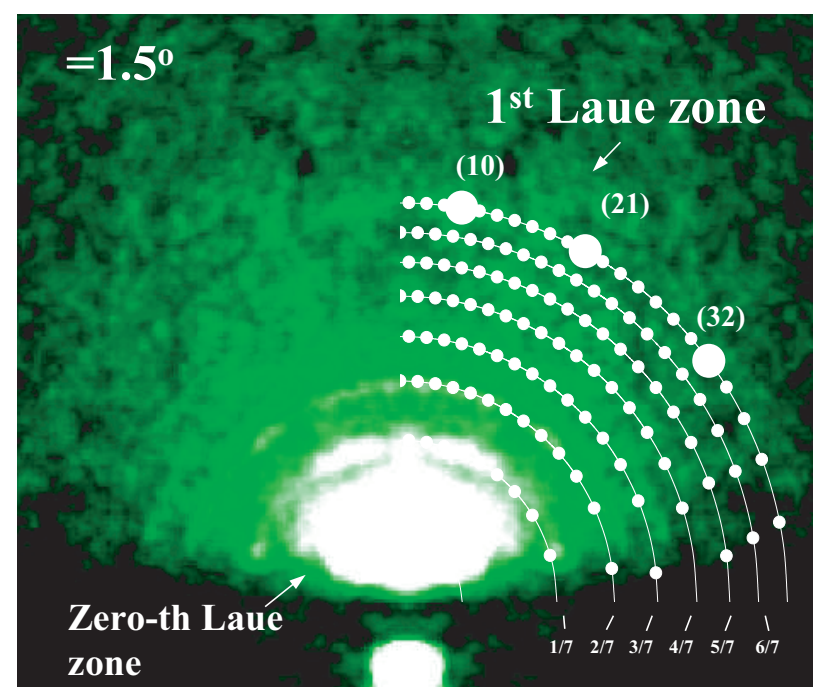

FIG. 2: Reflection high-energy positron diffraction pattern from a clean $\operatorname{Si}(111) 7 \times 7$ surface at the $[11 \overline{2}]$ incidence and at a glancing angle of $1.5^{\circ}$. Spot positions expected from the reciprocal lattice are also drawn in the right half part of the figure.

RHEPD approach. We used phosphorus-doped n-type $\mathrm{Si}(111)$ with a dimension of $15 \times 5 \times 0.5 \mathrm{~mm}$ and a resistivity of $10 \Omega \mathrm{cm}$. After surface cleaning with an acetone and ultra-pure water, the sample was transferred into a vacuum chamber evacuated to a base pressure of $5 \times 10^{-8}$ $\mathrm{Pa}$. The sample flashing was taken place by the direct current flow $(\sim 13 \mathrm{~A})$ for a short second and by cooling slowly to avoid the surface defect formation.

Figure 2 shows the diffraction pattern obtained at $\theta=1.5^{\circ}$ and at $[11 \overline{2}]$ incidence. This glancing angle nearly satisfies the condition of the first Bragg reflection. Three fractional order diffraction patterns are seen between the zero-th and first Laue zones. To confirm that these diffraction patterns are related to the $7 \times 7$ reconstruction, the spot positions expected from the $7 \times 7$ reciprocal lattice are also drawn in the figure. From this, the patterns are assigned to $1 / 7$-th to $3 / 7$-th Laue zones. Although individual spots are not well separated because of the limitation of the MCPA resolution, it is seen that the spot intensity varies in the same Laue zone. This feature may be interpreted using a full dynamical calculation. The other fractional order Laue zones, i.e., 4/7th to $6 / 7$-th, are unfortunately not clearly seen. This is probably due to inadequate signal-to-noise ratio. The further attempts should be made to observe weak diffraction pattern. The intensities of the fractional order patterns rapidly decreased with increasing the glancing angle. At $\theta=4.0^{\circ}$, these were invisible.

Figure 3 (a) shows the rocking curve of the specular spot in the one beam condition (incident direction is $7.5^{\circ}$ off oriented from the $[11 \overline{2}]$ direction). The intensity monotonically increases from $\theta=0.5^{\circ}$ and reaches a maximum at $\theta=1.6^{\circ}$. Three peaks are seen at $\theta=2.2^{\circ}, 2.7^{\circ}$ and $3.4^{\circ}$. The critical angle of total reflection is given by $\theta=\arcsin \left(V_{0} / E\right)^{1 / 2}$, where $V_{0}$ is the average crystal potential. Taking $V_{0}=12 \mathrm{eV}$, the critical angle of total reflection is $\theta=1.4^{\circ}$. The Bragg condition is given by $E \sin ^{2} \theta=37.5 n^{2} / d^{2}+V_{0}(n$ : integer, $d$ : bilayer dis- 


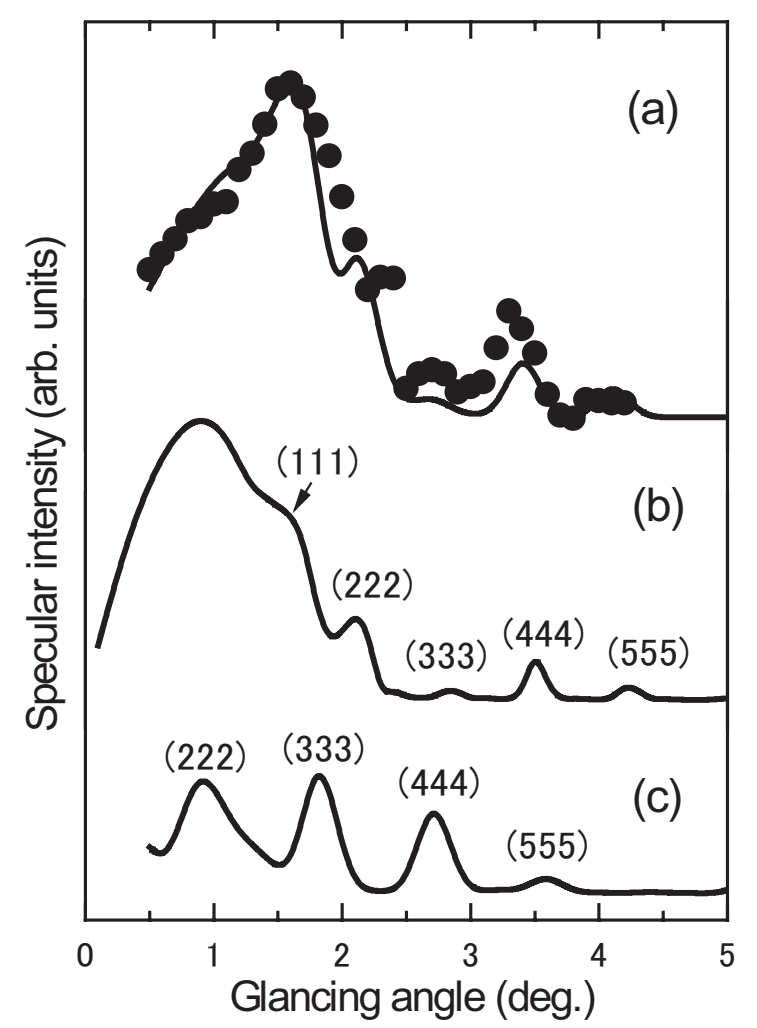

FIG. 3: (a) Filled circle represents the experimental rocking curve of specularly reflected positrons obtained from the clean $\mathrm{Si}(111) 7 \times 7$ surface at the one-beam condition $\left(7.5^{\circ}\right.$-off oriented from the $[11 \overline{2}]$ direction). Solid line is the best theoretical rocking curve to reproduce the experiment. (b) Rocking curve of specularly reflected positrons for $\operatorname{Si}(111) 7 \times 7$ surface calculated using the atomic configurations and absorption potentials determined in the previous RHEED study. (c) Rocking curve of specularly reflected electrons calculated using the same condition as (b).

tance $=3.14 \AA$ ). Thus, the region up to $1.4^{\circ}$ corresponds to total reflection. The peaks at $1.6^{\circ}, 2.2^{\circ}, 2.7^{\circ}$ and $3.4^{\circ}$ are assigned to the (111), (222), (333) and (444) Bragg reflections, respectively. The intensity distribution at $\theta<1.8^{\circ}$ reflects the condition of the topmost surface. No major intensity losses and dip structures are found below $1.6^{\circ}$. This indicates that the surface is atomically smooth. However, the slope of the curve changes at around $1.1^{\circ}$. This small discontinuity might be arising from the adatoms. Figure 3(c) shows the RHEED rocking curve at the beam energy of $20 \mathrm{keV}$ calculated with the optimized atomic configuration in the past RHEED study [10]. It is clearly seen that no total reflection occurs in the case of electrons and the (111) Bragg reflection is also absent. The higher order Bragg reflections are less sensitive to the topmost surface structure. These demonstrates that RHEPD is well suited for the surface structural analysis.

We attempted to reproduce the experimental rocking curve based on the dynamical calculation [13] and to extract the vertical position of adatoms. In the calculation, the crystal potential is expressed as a complex potential $\left(=V_{0}+i V^{\prime}\right)$. The imaginary part describes the absorp-

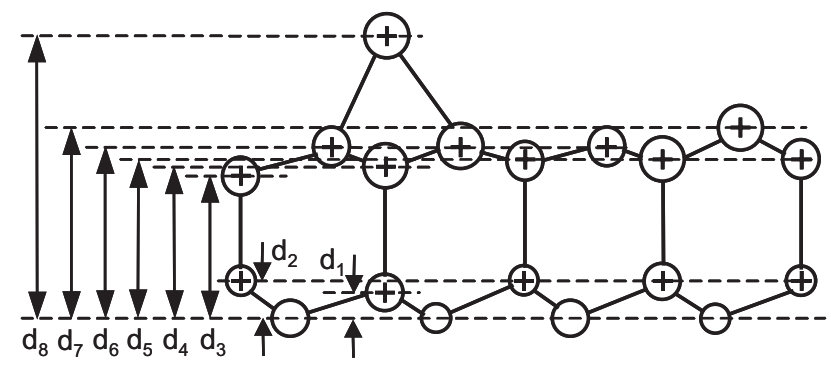

FIG. 4: Definition of interlayer distances of $\mathrm{Si}(111) 7 \times 7$ surface.

TABLE II: Mean distance between adatom and stacking fault layers $\left(d_{a d}\right)$ determined in the present and previous studies.

\begin{tabular}{|c|c|c|c|c|}
\hline Present & $\mathrm{XRD}[7]$ & RHEED $[5,6]$ & LEED [4] & Theory [8] \\
\hline $1.52 \AA$ & $1.58 \AA$ & 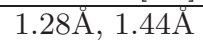 & $1.23 \AA$ & $1.34 \AA$ \\
\hline
\end{tabular}

tion through the inelastic processes, which is composed of terms related to phonon scattering $\left(V_{p h}\right)$ and the electronic excitation $\left(V_{e l}\right)$. Thermal vibration of atoms is represented by the Debye-Waller factor. The layer sequences in the DAS structure are assumed as shown in Fig. 4.

At first, the rocking curves of specularly reflected positrons were calculated using the atomic configurations and absorption potential determined in the RHEED study [10]. Figures 3(b) shows thus calculated rocking curve. It is readily seen that the calculation is compatible to the experiment at $\theta>2.0^{\circ}$ but not in the total reflection region and around the first Bragg peak $\left(\sim 1.6^{\circ}\right)$. This is because in the RHEED the calculation conditions are optimized so as to reproduce the features of the higher order Bragg reflections. To reproduce the experimental rocking curve, the interlayer distances, the absorption potential and the Debye-Waller factor used in the RHEED study should be modified. During the preliminary calculations, we found that the rocking curve is quite sensitive to the relative distance between adatom and stacking fault layers $\left(d_{a d}=d_{8}-d_{6}\right)$. It is also found that the changes of the Debye-Waller factor and $V_{p h}$ have only minor effects on the curve shape in the total reflection region. Thus, the distance between the adatom and stacking fault layers and the absorption potential due to electronic excitations $\left(V_{e l}\right)$ are varied so as to reproduce the experimental rocking curve. The Debye-Waller factor and $V_{p h}$ are fixed at $0.3 \AA^{2}$ and $0.2 \mathrm{~V}$, respectively. The solid line in Fig. 3 (a) is the calculated best rocking curve. The optimum distance between adatom and stacking fault layers was determined to be $d_{a d}=1.52 \AA$. Also, we obtained $V_{e l}=0.25$ $\mathrm{V}$. The Pendry R-factor is estimated to be 0.17 suggesting that the experimental curve is well reproduced. Table II lists the $\mathrm{d}_{a d}$ values obtained in the present and previous studies.

Early electron diffraction studies suggest $d_{a d}=1.23-1.28$ $\AA[9,10]$, which is comparable to the result from the first principles calculation $\left(d_{a d}=1.34 \AA\right)$ [14]. Recent refined RHEED study [11] reported a greater value $\left(d_{a d}=1.44 \AA\right)$. The XRD study gives a remarkably high value $\left(d_{a d}=1.58\right.$ $\AA)$ [12]. The present value $\left(d_{a d}=1.52 \AA\right)$ is in the middle between these values. It has long been thought that 


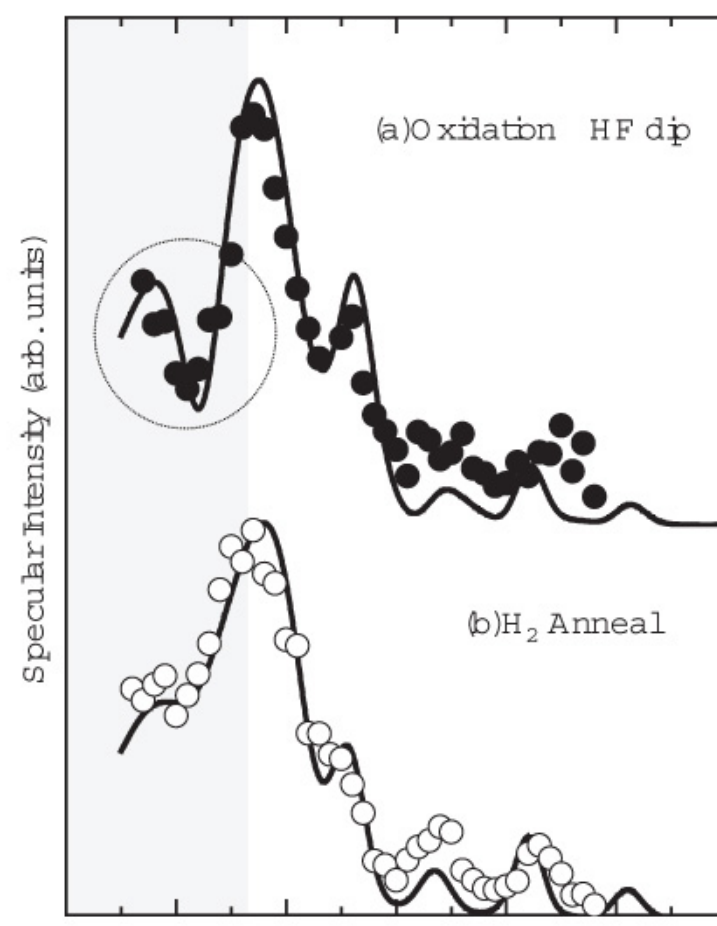

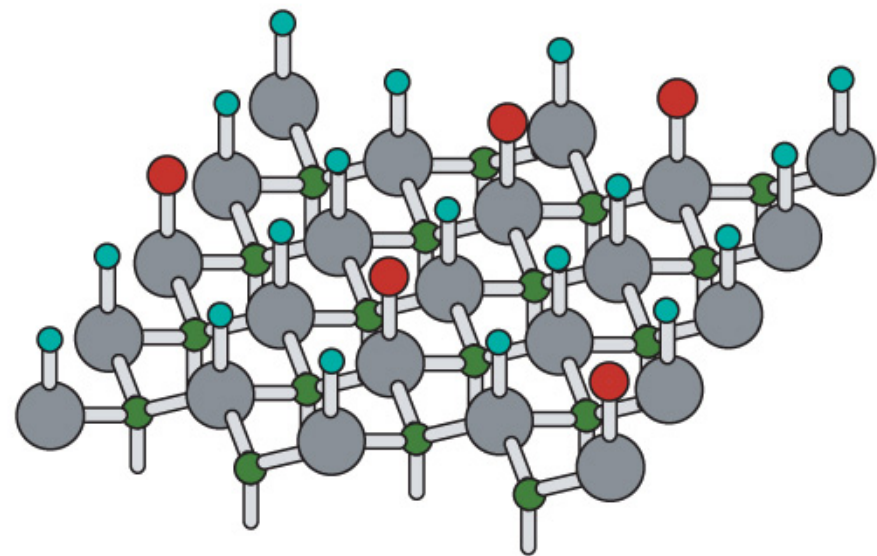

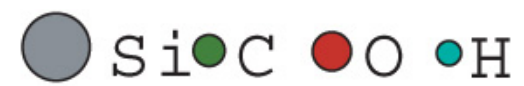

(C)

G lancing Angle (deg.)

FIG. 5: Rocking curves of specular reflection spot obtained from $\mathrm{SiC}(0001)$ surfaces (a) after the sacrificial oxidation and subsequent $\mathrm{HF}$ dipping and (b) after hydrogen etching at $1450^{\circ} \mathrm{C}$ for 2 hours at one-beam condition $\left(7.5^{\circ}\right.$-off oriented from ). Solid lines represent calculated curves by the dynamical diffraction theory assuming the atomic structure schematically represented in (c).

the XRD overestimated $d_{a d}$. However, the above results indicate that $\mathrm{d}_{a d}$ determined in the XRD study is not necessarily unrealistic. Thus, the distance of the adatom and stacking fault layers should be around $1.5 \AA$. The first principles calculations underestimate $\mathrm{d}_{a d}$ and hence the improvement of the theory is needed.

The absorption potential due to the electronic excitation obtained above is approximately a half of that anticipated from the RHEED study [10] and a theory considering the bulk plasmon excitation [15]. Since the cross section of plasmon excitation itself should be quite similar for electrons and positrons, the smaller absorption potential may be interpreted as a lack of bulk plasmon excitation due to incident positrons. Probably, incident positrons are reflected mostly at the topmost surface and hence the surface plasmon excitation with a smaller energy may be more efficient as compared to the bulk plasmon excitation.

\section{B. $\mathrm{SiC}(0001)$}

$\mathrm{SiC}$ is a promising semiconductor for high power and high temperature device applications. However, because of its high chemical and mechanical stabilities the surface polishing is rather problematic. As a consequence, the commercial surface is generally quite rough even after the etching treatment. This inhibits the growth of high quality epitaxial layer and the fabrication of devices. In this study, we examined three treatments to create atomically flat surface; (i) sacrificial oxidation in a dry oxygen ambient at $1200^{\circ} \mathrm{C}$ for 4 hours and $\mathrm{HF}$ (50\%) dipping [16], (ii) exsitu hydrogen etching (pressure: 100 Torr) at $1400^{\circ} \mathrm{C}$ for 8 hours [17] and (iii) infrared lamp flashing at $1200^{\circ} \mathrm{C}$ in an ultra high vacuum. We used the modified Lely-grown $6 \mathrm{H} \mathrm{SiC}$ doped with nitrogen.

After the sacrificial oxidation and HF dipping, the RHEPD pattern exhibit the clear $1 \times 1$ periodicity suggesting no super structure was formed. Figure 5(a) shows the rocking curves of specular reflection spot.

Since the crystal potential for $\mathrm{SiC}$ is approximately 17 $\mathrm{eV}$ for $\mathrm{SiC}$, the total reflection region should be below $1.7^{\circ}$. Observed peaks at $1.8^{\circ}, 2.5^{\circ}, 3.3^{\circ}$ and $4.2^{\circ}$ are assigned to the first to fourth Bragg reflections using the Bragg equation $\left(E \sin 2 \theta=37.5 n^{2} / d^{2}+V_{0}\right.$ with $d=2.56$ $\AA)$. A distinct absorption peak is seen in the total reflection region (at $\theta=1^{\circ}$ ). This is the typical example of the interference and inelastic effect of positron beams because of the double potential at the surface. It is reported that after the HF dipping a part of surface dangling bonds is terminated with atomic hydrogen while the rest dangling bonds with oxygen atoms and or hydroxyl species [17]. As shown in Fig. 6, the oxygen peak $\left(\mathrm{O}_{K L L}\right)$ was detected by the AES measurements. Therefore, the above dip is likely to be due to oxygen and/or hydroxyl adsorptions. The rocking curve was then calculated assuming the attachment of oxygen atoms at the $\mathrm{T} 1$ site and using the dynamical diffraction theory. The solid line shown in the figure is the calculated rocking curve with an oxygen coverage of $50 \%$ and the bond length between the first layer Si atoms to be $1.88 \AA$. The Pendry R-factor 


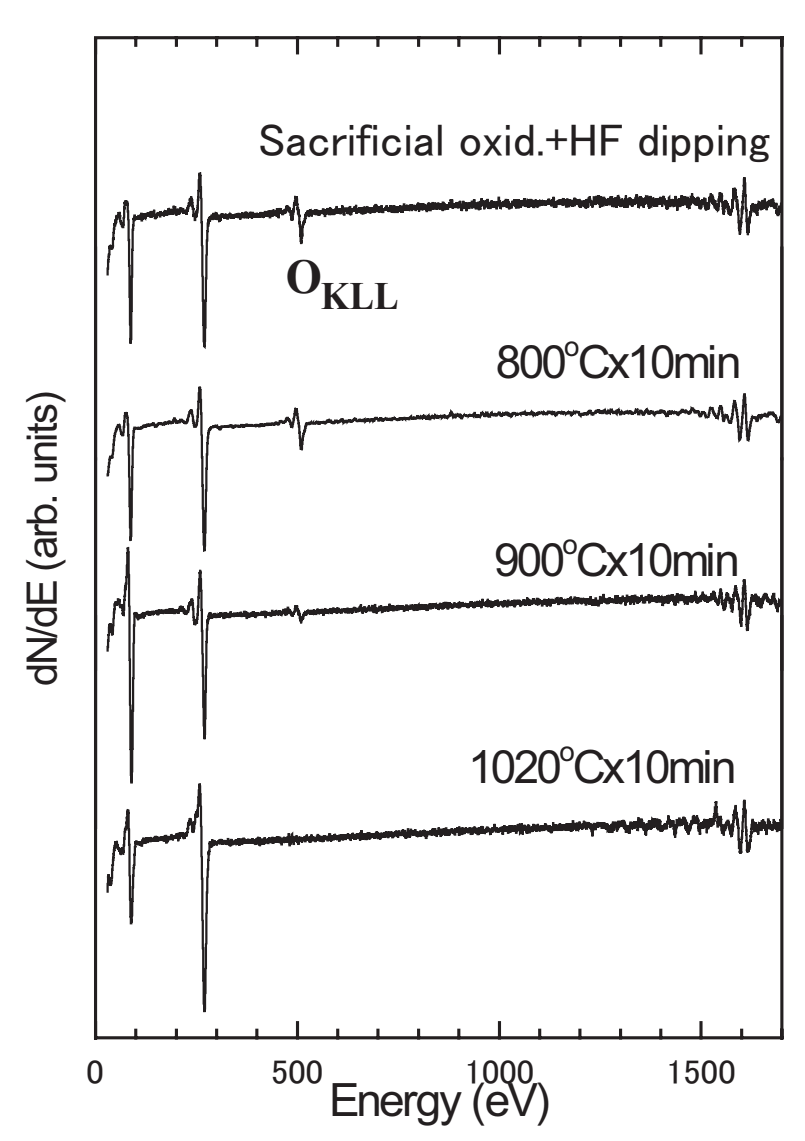

FIG. 6: Auger electron spectroscopy spectra obtained after sacrificial oxidation, HF dipping and flashing by an infrared lamp in UHV.

was 0.15 suggesting that the experimental rocking curve is well reproduced with the above assumption. The bond length is $0.2 \AA$ greater than that obtained in the previous LEED experiments. Such a discrepancy is also seen in the above analysis of the adatoms on the $\operatorname{Si}(111) 7 \times 7$ surface. It seems that LEED tends to underestimate the bond lengths. As for the oxygen-terminated surfaces, scanning tunneling microscopy is hardly applied because of the inconveniently weak tunneling current. Only diffraction technique is available to determine the atomic configuration.

The diffraction pattern represented again a $1 \times 1$ periodicity after the high temperature hydrogen etching. This suggests that hydrides discretely distributes on the surface. The absorption peak in the total reflection region observed in Fig. 5(a) no longer appears in the total reflection region as shown in Fig. 5(b). This indicates that the oxygen adsorption is far suppressed from the HF-dipped state. However, a small plateau is still seen at $0.5-1.0^{\circ}$ region. Although the surface dangling bonds are though to be terminated with atomic hydrogen much more completely as compared to the HF dipping [18], the AES measurement revealed the adsorption of oxygen atoms. The plateau may be explained considering oxygen. The solid line in the figure represents the best rocking curve calculated with the dynamical diffraction theory assuming the oxygen coverage of $10 \%$. Thus, even after the hydrogen etching initial oxidation proceeds. Probably, the oxygen adsorption occurs during cool-down after the hydrogen etching and/or after the exposure to the air. It is reported that upon hydrogen etching under an atmospheric pressure the $\mathrm{SiC}(0001) \sqrt{3} \times \sqrt{3}-\mathrm{R} 30^{\circ}$ structure appears [19]. This reconstructed surface is explained as the silicate mono-layer. In this study, however, no such a structure was observed.

Thus, the above two methods are not perfect to obtain an atomically flat surface. As shown in Fig. 6, oxygen atoms remaining after the $\mathrm{HF}$ dipping can be removed by the flashing above $900^{\circ} \mathrm{C}$ in an ultra-high vacuum. After flashing at $1020^{\circ} \mathrm{C}$, the carbon peak $\left(\mathrm{C}_{K L L}\right)$ becomes greater than the silicon peak $\left(\mathrm{Si}_{L V V}\right)$. Yet, no thick graphite layer is formed. In the carbon peak, a chemical shift to the graphite phase is also seen. In this flashing condition, the $6 \sqrt{3} \times 6 \sqrt{3}$-like diffraction pattern is frequently observed [21]. This structure was however explained as not real super structure but as double scattering due to the graphite monocrystal. In this study, the RHEPD pattern exhibited a $1 \times 1$ periodicity. The diffraction pattern from the graphite monocrystal was hardly observed probably because of its weak intensity.

Figure 7 shows the rocking curve obtained after the flashing at $1020{ }^{\circ} \mathrm{C}$. The absorption peak in the total reflection region observed after the HF dipping completely vanishes. This means that by flashing an oxygen free and atomically flat surface can be fabricated. Considering the above AES measurements, the occurrence of the flat surface is assisted by the surface graphitization [20]. If the graphite layer exists on the $\mathrm{SiC}(0001)$ surface, a dip structure is likely to appear in the rocking curve of totally reflected positrons because of the double reflection of the positron beam at the graphitic and $\mathrm{SiC}$ layers. However, no apparent dip structure is seen below the critical angle in the rocking curve shown in Fig. 7. From the calculation based on the dynamical diffraction theory, it was found that the dip structure appears when the coverage of the graphite layer is greater than one. The solid line in Fig. 7 represents the calculated rocking curve with the graphite coverage of $0.7 \mathrm{ML}$ and the interlayer distance of $3.2 \AA$. The interlayer distance is nearly the same as that for the graphite crystal. Thus, it is concluded that the graphite layer formed on the $\mathrm{SiC}(0001)$ is fairly weakly bound on it because of the van der Waals force.

\section{SUMMARY}

We have developed an electrostatic positron beam suited for RHEPD experiments. The beam coherence length is long enough to observe large surface super structures. Indeed, we succeeded in observing a RHEPD pattern from the clean $\mathrm{Si}(111) 7 \times 7$ reconstructed surface. The rocking curve of the specular spot suggests that the adatom vertical position relative to the first layer is sufficiently shifted towards the vacuum region than the expectation from the electron diffraction. The previous adatom vertical position determined using XRD is not necessarily overestimated. We also applied RHEPD technique to the analyses of $\mathrm{SiC}(0001)$ surfaces. After the sacrificial oxidation and HF dipping, an absorption peak was seen in the total reflection region. This was explained in terms of the 


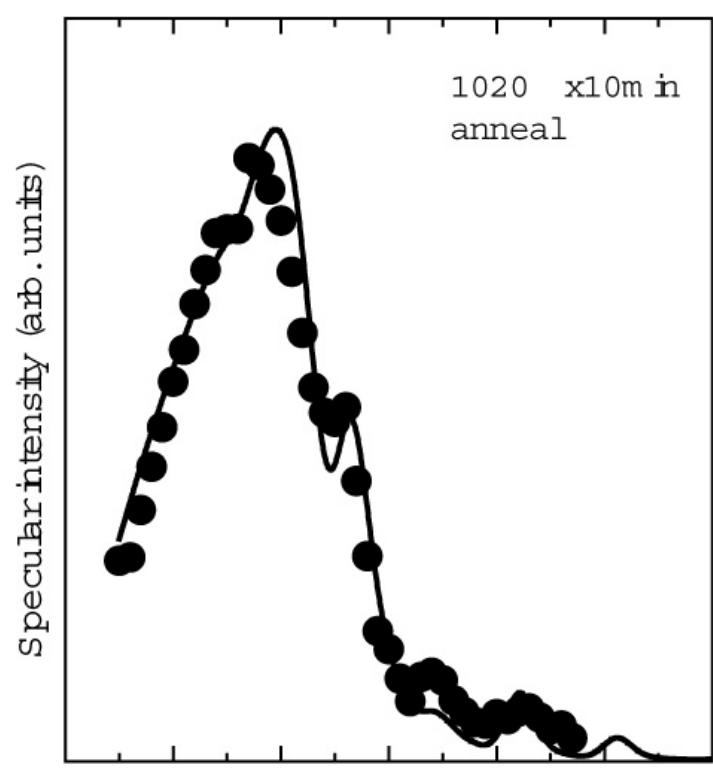

G lancing angle (deg.)

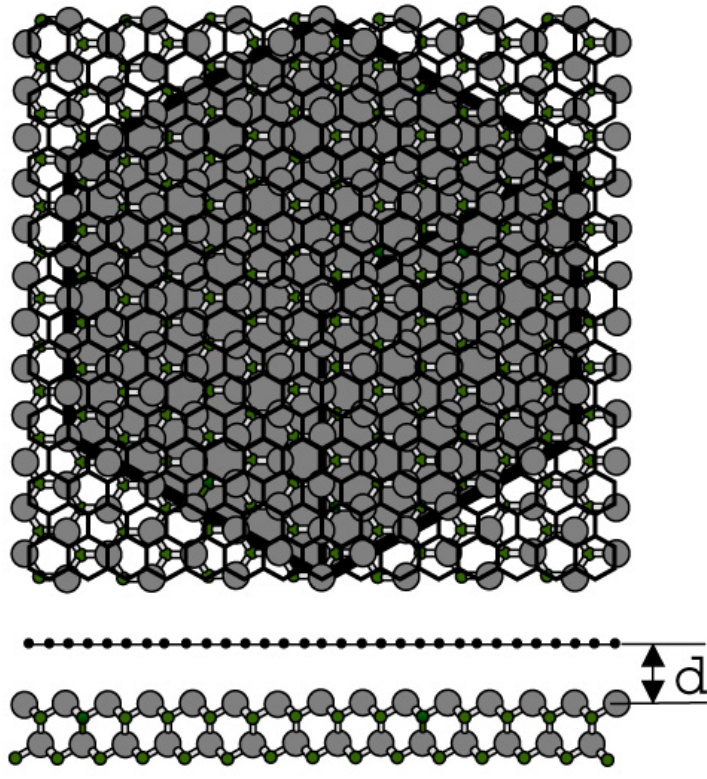

Sioc ? Traphite

(a)

(b)

FIG. 7: (a) Rocking curve of specular reflection obtained from the $\mathrm{SiC}(0001)$ surface after sacrificial oxidation, HF dipping and flashing by an infrared lamp in UHV at one-beam condition $\left(7.5^{\circ}\right.$-off oriented from $)$. Solid line represents the calculated curve by dynamical diffraction theory assuming $0.7 \mathrm{ML}$ graphite on the $\mathrm{SiC}(0001)$ surface shown in (b).

interference and inelastic effects due to oxygen adsorption. It was found that the initial oxidation already proceeded after the high temperature hydrogen etching. An oxygenfree and atomically flat SiC surface was obtained accompanying the graphitization by flashing above $1000^{\circ} \mathrm{C}$ in an ultra high vacuum. The interlayer distance between the graphite layer and the substrate was estimated to be comparable to that for graphite crystal. The above studies demonstrate that RHEPD technique is useful in the structural analysis of the topmost surfaces.

\section{Acknowledgments}

This work was partly promoted as the Nuclear Energy Fundamentals Crossover Research in the Ministry of Education, Culture, Sports, Science and Technology of Japan.
[1] A. Ichimiya, Solid State Commun. 28-29, 143(1992).

[2] A. Kawasuso, S. Okada, and A. Ichimiya, Nucl. Instrum. Methods Phys. Res. B 171, 219(2000).

[3] T. Ishimoto, A. Kawasuso, and H. Itoh, Appl. Surf. Sci. 194, 43(2002).

[4] K. F. Canter, P. H. Lippel, W. S. Crane and A. P. Mills, Jr., in: Positron Studies of Solids, Surfaces, and Atoms, eds. A. P. Mills, Jr., W. S. Crane and K. F. Canter (World Scientific, Singapore, 1986) p. 199.

[5] G. R. Brandes, K. F. Canter, T. N. Horsky, P. H. Lippel and A. P. Mills, J. Phys. Condens. Matter 1, SA135(1989).

[6] G. Comsa, Surf. Sci. 81, 57(1979).

[7] K. Takayanagi, Y. Tanishiro, S. Takahashi, and M. Takahashi, Surf. Sci. 164,367(1985).

[8] G. Binnig, H. Rohrer, Ch. Gerber, and E. Weibel, Phys. Rev. Lett. 50, 120(1983).

[9] H. Huang, S. Y. Tong, W. E. Packard, and M. B. Webb, Phys. Lett. A 130,166(1988).

[10] A. Ichimiya, Surf. Sci. 192, L893 (1987).
[11] Y. Fukaya, K. Nakamura, and Y. Shigeta, J. Vac. Sci. Technol. A 18,968(2000).

[12] I. K. Robinson and E. Vlieg, Surf. Sci. 261,123(1992).

[13] A. Ichimiya, Jpn. J. Appl. Phys., Part 2, 22, 176(1983).

[14] Karl D. Brommer, M. Needels, B. E. Larson, and J. D. Joannopoulos, Phys. Rev. Lett. 68,1355 (1992).

[15] G. Radi, Acata Cryst. A 26, 41 (1970).

[16] A. Kawasuso, K. Kojima, M. Yoshikawa, H. Itoh and K. Narumi, Appl. Phys. Lett. 76, 1119 (2000).

[17] U. Starke, Phys. Stat. Sol. (b)202, 475 (1997).

[18] H. Tsuchida, I. Kamata and K. Izumi, Jpn. J. Appl. Phys. Part 2, 36, L699 (1997).

[19] J. Bernhardt, J. Shardt, U. Starke and K. Heinz, Appl. Phys. Lett. 74, 1084 (1999).

[20] Y. Hisada, K. Hayashi, K. Kato, T. Aoyama, S. Mukainakano and A. Ichimiya, Jpn. J. Appl. Phys. Part 1, 40,2211(2001).

[21] A. J. van Bommel, J. E. Clombeen and A.van Tooren, Surf. Sci. 48, 463(1975). 